\title{
Parkinsonism with Neuroacanthocytosis
}

\author{
R.F. Peppard, C.S. Lu, Nai-Shin Chu, P. Teal, W.R.W. Martin and D.B. Calne
}

\begin{abstract}
Two patients with neuroacanthocytosis are described. One presented with parkinsonism and the other resembled diurnal dystonia of the Segawa type. Both patients responded well to dopaminomimetic therapy. A PET scan with fluorodopa revealed a nigrostriatal deficit in the first patient.
\end{abstract}

RÉSUMÉ: Parkinsonisme et neuro-acanthocytose Nous décrivons deux patients avec neuro-acanthocytose. Un des patients s'est présenté avec du parkinsonisme et l'autre avec une dystonie diurne de type Segawa. Les deux patients ont bien répondu à la thérapie dopaminomimétique. Un PET scan à la fluorodopa a révélé un déficit nigrostrié chez le premier patient.

Can. J. Neurol. Sci. 1990; 17:298-30I

Acanthocytes are abnormal red blood cells characterized by thorny projections which are evident in wet film preparations. This red blood cell change has been associated with a variety of hereditary neurological conditions. Bassen and Kornzweig 1 described the neurological disorder found with abetalipoproteinemia and Mars $^{2}$ et al reported the form of neuroacanthocytosis that occurs with hypobetalipoproteinemia. A syndrome which includes chorea, tics, dystonia, areflexia and distal amyotrophy has been associated with acanthocytosis and normal lipoprotein levels. $3,4,5$

Acanthocytes are mature erythrocytes with $3-12$ spicules as blunt projections arising with uneven distribution from the cell membrane. They must be differentiated from spur cells which are found in liver disease with hypercholesterolemia. Spur cells often have 10-30 evenly spaced spicules. ${ }^{6.7}$ Although originally described in association with abetalipoproteinemia, acanthocytes have also been detected in neurologic conditions with normal lipoproteins such as neuroacanthocytosis and with the McLeod phenotype of blood group antigens associated with late onset myopathy.

No universally accepted mechanism has been established for the formation of acanthocytes. In abetalipoproteinemia there is an abnormal distribution of membrane phospholipids with a deficiency of linoleic acid and a reversal of the phosphatidylcholine to sphingomyelin ratio. The membrane cholesterol content may be increased and changes have been noted in other membrane properties such as decreased fluidity and increased microviscosity. McLeod red cells have deficiencies in nonstructural membrane proteins which may alter membrane fluidity. In neuroacanthocytosis changes in membrane fluidity have been reported but cholesterol and phospholipid levels are normal. The most consistent disturbance that has been found in acanthocytosis is an increase in the proportion of sphingomyelin. ${ }^{8}$ The neuro- logic disorders associated with acanthocytosis are summarized in Table 1.

We describe two patients which extend the clinical description of neuroacanthocytosis with normal lipoproteins. Patient 1 had severe parkinsonism with associated mild distal neuropathy which appeared to be inherited in autosomal dominant fashion. The case is noteworthy for the absence of hyperkinesias (other than those induced by levodopa) and for the evidence of striatal dopamine depletion using positron emission tomography with [ $\left.{ }^{18} \mathrm{~F}\right]-6$-fluoro-L-dopa. The second patient presented with diurnally fluctuating dystonia and parkinsonism. Both cases responded to levodopa therapy.

\section{Case Reports}

\section{Patient I}

This 47 year black male from Antigua developed resting tremor in his hands 14 years ago. The tremor gradually became more severe and spread to involve his feet. He noticed leg stiffness with some impairment in his walking and in getting up from a chair. Six years ago he developed postural instability and short stepping gait with occasional freezing and falls. For the last two years he has usually required support when walking and has been increasingly troubled by falls. He has recently developed numbness and painful, burning paresthesiae in the feet and legs extending up to the knees. When first seen he was taking Sinemet 250/25 half tablet four times daily and benztropine $2 \mathrm{mg}$ qid.

Family history revealed that his brother, father, two paternal uncles and paternal grandfather had all suffered from a similar progressive neurological illness. None of his six sisters, his only paternal aunt or paternal grandmother have developed this condition.

On examination, mental state was normal. There was masking of facial expression and hypophonia. He had intermittent torticollis and dystonia of the lower face. At different times his limbs displayed dystonia, chorea or tremor. There was mild wasting of the leg muscles below the knees and mild bilateral weakness of eversion and dorsiflexion of the ankle and extension of the big toe. Occasional fasciculations were observed in the calf muscles. Mild rigidity was present in the legs and cogwheeling was detected at the left wrist with reinforcement. Tendon

From the Department of Medicine, University of British Columbia, Vancouver (R.F.P., P.T., W.R.W.M., D.B.C.)

and Department of Neurology, Chang Gung Memorial Hospital, Taipei, Taiwan, R.O.C. (C.S.L., N.-S.C.)

Received October 18, 1989. Accepted in final form May 2, 1990

Reprint requests to: Dr. D. Calne, Division of Neurology, University Hospital, 2211 Wesbrook Mall, U.B.C., Vancouver, British Columbia, Canada V6T IW5 
reflexes were diminished in the upper limbs; the knee and ankle jerks were absent. Plantar responses were flexor. Sensation was normal. He was unable to stand heel to toe but could stand with his feet together without assistance. His gait was slow, shuffling and unsteady with decreased arm swing. When medication was withdrawn, the choreatic and dystonic movements ceased but tremor and bradykinesia became more prominent and he was unable to walk independently. The subsequent introduction of bromocriptine led to a smoother response with decreased dyskinesias

The standard peripheral blood film was normal but immediate examination of a wet preparation revealed $4 \%$ acanthocytes (Figure 2). Lipoprotein electrophoresis revealed normal HDL cholesterol, triglycerides, HDL, Apoprotein A. Cholesterol was $310 \mathrm{mg} / \mathrm{dl}$ (normal 120280 ), LDL was $229 \mathrm{mg} / \mathrm{dl}$ (normal 104-186), Apoprotein B was $137 \mathrm{mg} / \mathrm{dl}$ (normal 67-117). Electrophysiological studies were consistent with a predominantly axonal sensory polyneuropathy. PET with 618F-fluoro-L-dopa showed marked bilateral reduction in uptake similar to that seen with severe idiopathic parkinsonism (Figure 1).

\section{Patient 2}

A 21 year Chinese man developed dystonia of the right foot five years ago. he also became aware of stiffness and slowness of movement in his right leg. Progressive inversion of the right foot occurred. Four years ago he developed resting tremor of the right leg. The tremor, stiff-

\section{Table 1: Neurologic Disorders Associated with Acanthocytosis}

\begin{tabular}{|c|c|}
\hline Disorder & Serum \\
\hline Bassen-Kornzweig & $\begin{array}{l}\text { abetalipoprotein, low cholesterol, } \\
\text { low triglycerides. }\end{array}$ \\
\hline Familial hypobetaliproteinemia & $\begin{array}{l}\text { low betalipoproteins, low } \\
\text { cholesterol, low triglycerides. }\end{array}$ \\
\hline $\begin{array}{l}\text { Neuroacanthocytosis } \\
\text { McLeod phenotype }\end{array}$ & $\begin{array}{l}\text { normal serum lipids and lipoproteins. } \\
\text { normal serum lipids and lipoproteins. } \\
\text { elevated creatine kinase. }\end{array}$ \\
\hline
\end{tabular}

Rare sporadic cases of acanthocytosis have been reported in Hallervorden-Spatz disease and mitochondrial encephalomyopathy. ${ }^{10}$

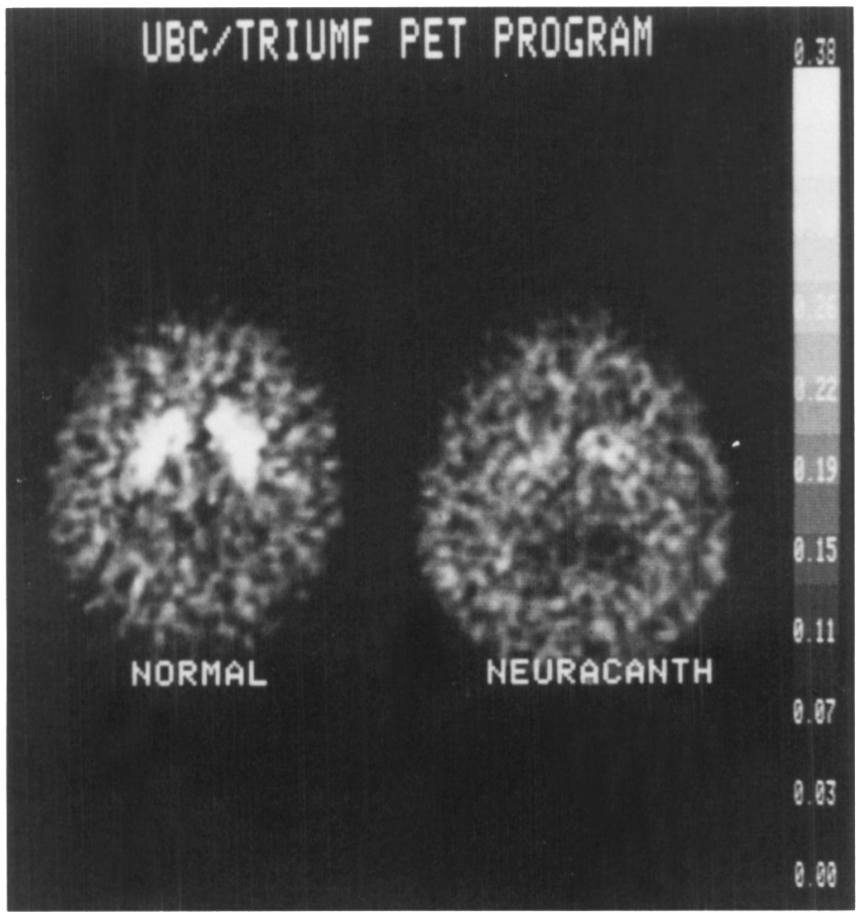

Figure I $-{ }^{18}$ F-dopa scans (axial views through the basal ganglia). On the right is Case 1 , and on the left a comparable view from a normal subject of similar age. ness and slowness of movement progressed to involve the whole body. Facial expression diminished and his voice became soft. Gait steadily deteriorated and he began to fall. Severe depression evolved. There was no family history of parkinsonism or of consanguinity.

On examination, cognitive function was normal. There was hypomimia and hypophonia. The right foot was held in an equinovarus position with dorsiflexion of the great toe and plantar flexion of the other toes. Prominent resting tremor was present in the left arm and leg. Cogwheel rigidity was evident in all limbs. Rapid alternating movements of the hands and feet were poorly performed. The deep tendon reflexes were brisk but the plantar responses were flexor. No muscle wasting or weakness was present and sensory examination was normal. He had a stooped posture. His gait displayed slow initiation, festination, propulsion, and unsteadiness with reduced arm swing.

On repeated wet smears of peripheral blood, 6-12\% acanthocytes were found (Figure 2). Serum cholesterol, triglycerides and lipoprotein electrophoresis were normal. EMG and nerve conduction were normal. Both the parkinsonism and the dystonia responded well to bromocriptine $30 \mathrm{mg}$ per day and levodopa $250 \mathrm{mg}$ with carbidopa $25 \mathrm{mg}$ per day. He was able to walk normally for the first time in four years and resumed employment in a pottery factory.

\section{Discussion}

In patient 1 , the family history of a progressive neurological disorder with onset in the third decade of life and the clinical

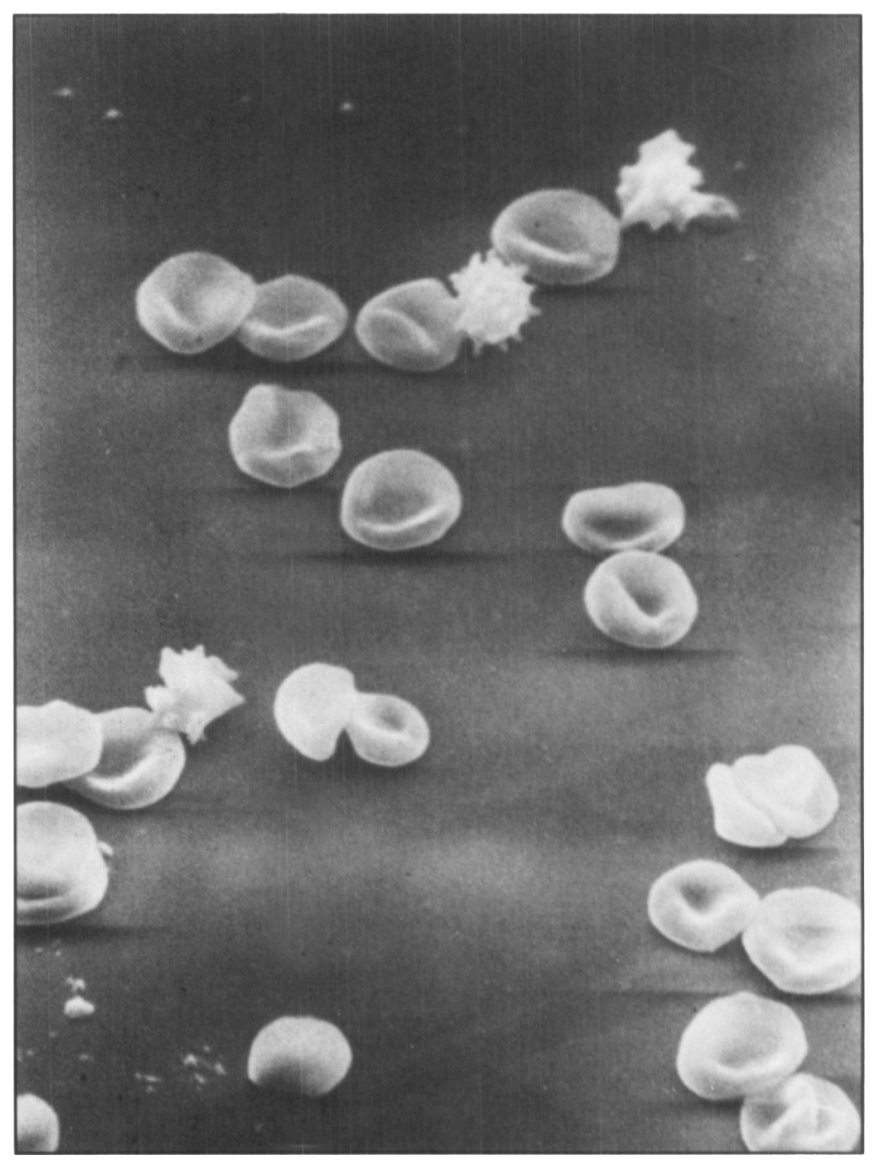

Figure 2 -lllustration of the erythrocytes from Case 2 Magnification $\times$ 3000. A few drops of blood obtained by venepuncture were immediately placed into the fixative containing $10 \mathrm{ml}$ of $3 \%$ glutaraldehyde in 0.I M phosphate buffer, $p H$ 7.4. After settling for 6 hours, red cells were washed in $0.9 \% \mathrm{NaCl}$ and then dehyrated in a graded series of ethanol. After being coated with platinum-palladium, the red cells were viewed at $15 \mathrm{kV}$ in a scanning electron microscope (Hitachi S-450) 
picture of severe parkinsonism with associated distal amyotrophy in the lower limbs made the diagnosis of idiopathic parkinsonism unlikely and neuroacanthocytosis was suspected. It was not clear, initially, whether the dyskinesia was related to his disease or his treatment, but the suspension of levodopa therapy demonstrated that the hyperkinetic features were drug-induced.

Autosomal recessive and autosomal dominant patterns of inheritance have been described in neuroacanthocytosis with normal lipoproteins. Autosomal dominant inheritance was most likely in the first families which were reported with this condition ${ }^{3.5}$ but subsequent reports have suggested that autosomal recessive inheritance is more common. 11 It was considered that the pattern of inheritance in the family of patient 1 was most consistent with autosomal dominant inheritance. The failure of transmission to any females in this family was, in all likelihood, a coincidence. Some possibility must remain that females with the abnormal gene are in some way protected from developing the disease in this family. Because the family lives in the West Indies, examination of other members was limited to two of the proband's children. Both had generalized areflexia but without other neurological abnormalities. Only one child had a wet blood smear examination and the appearance of the red blood cells was entirely normal.

In patient 2, there was no family history. Onset was with focal dystonia involving one foot, which progressed to severe parkinsonism with rest and action tremor. A dramatic response to levodopa therapy was seen.

The normal or elevated betalipoproteins in these two patients exclude those forms of neuroacanthocytosis which are associated with hypobetalipoproteinemia ${ }^{2}$ or with abetalipoproteinemia. ${ }^{1}$ Kell blood group was negative but Cellano positive which excludes MacLeod's syndrome, another cause of acanthocytosis with associated neurological manifestations. 12

These two patients represent forms of "neuroacanthocytosis with normal lipoproteins". It has been suggested that the forms of neuroacanthocytosis in which normal levels of lipoproteins are found should be categorized according to the major neurological manifestations. ${ }^{13}$ The variety of clinical features seen has been reviewed. $4,11,14,15,16$

Chorea is often prominent in this disorder, leading to the earlier designation "choreoacanthocytosis". Perioral and facial hyperkinesias are frequent early manifestations and may result in injury to the lips and tongue. Chorea subsequently involves the upper limbs and, later, the legs. Dystonia, tics and involuntary vocalizations have been described. Other manifestations which have been reported include progressive muscular atrophy, areflexia, peripheral neuropathy, ataxia, mental deterioration and epilepsy. 17.18

Although patients in whom chorea, tics and dystonia have given way to or coexisted with parkinsonism, have been described, ${ }^{13.15}$ patient 1 is unusual in manifesting as parkinsonism without hyperkinetic features. The combination of acanthocytosis, tics, motor neuron disease and progressive parkinsonism has been seen in two brothers of consanguineous parents. ${ }^{19}$ In these patients orofacial movements and vocalizations lessened or resolved as parkinsonian features developed. Despite cerebrospinal fluid homovanillic acid levels which suggested impairment of pre-synaptic dopaminergic function in one patient, there was no response to levodopa.
The nature of the disorder in family members of patient 1 rests on the history. It is not possible to say with certainly that the index patient did not have hyperkinetic manifestations early in the course of his disease. However the description of symptoms, the previous diagnosis of idiopathic parkinsonism and the favorable response to levodopa over many years make it unlikely that a hyperkinetic state was overlooked. The drug-induced dyskinesias only developed after several years of antiparkinson therapy.

Patient 2 resembles the diurnally fluctuating hereditary progressive dystonia described by Segawa et al, 20 in that deficits disappeared following sleep, and became worse during the course of the day, with a response to levodopa therapy. Patient 2 is also noteworthy for the prominence of parkinsonian features from early in the course of the illness.

The pathological lesions in neuroacanthocytosis are striatal atrophy with neuronal loss and gliosis. There has been no description of degeneration of the substantia nigra in autopsied patients with neuroacanthocytosis. 21,22 However, in a report of the pathology of chronic spherocytic hemolytic anemia with a similar clinical syndrome of chorea-amyotrophy, neuronal loss in the substantia nigra has been noted. 23

These patients demonstrate that neuroacanthocytosis with normal lipoproteins can present with severe parkinsonism. In vivo evidence of nigrostriatal damage was provided by the fluorodopa scan in patient 1 . The responsiveness to levodopa and bromocriptine in both these patients also supports the presence of a pre-synaptic defect in the nigrostriatal dopaminergic system.

\section{ACKNOWLEDGEMENT}

We wish to thank Dr. C. Clark for his assistance in the analysis of the PET scans. This work was supported by the Dystonia Medical Research Foundation and the Medical Research Council of Canada.

\section{REFERENCES}

1. Bassen FA, Kornzweig AL. Malformation of the erythrocytes in a case of atypical retinitis pigmentosa. Blood 1950; 5: 381-387.

2. Mars H, Lewis LA, Robertson AL, et al. Familial hypolipoproteinemia: a genetic disorder of lipid metabolism with nervous system involvement. Am J Med 1969; 46: 886-900.

3. Critchley EMR, Clark DB, Wikler A. Acanthocytosis and neurological disorder without betalipoproteinemia. Ann Neurol 1968; 18: $134-140$.

4. Levine IM, Estes JW, Looney JM. Hereditary neurological disease with acanthocytosis. Arch Neurol 1968; 19: 403-409.

5. Aminoff MJ. Acanthocytosis and neurological disease. Brain 1972; 95: 749-760.

6. Williams WJ, Beutler E, Erslev AJ, et al. Hematology. 3rd Edition. McGraw-Hill 1983; 556-559.

7. Pittiglio DH, Sacher RA. Clinical hematology and fundamentals of hemostasis. F.A. Davis \& Co. 1987; 396-397.

8. Clark MR, Aminoff MJ, Chiu DT-Y, et al. Red cell deformability and lipid composition in two forms of acanthocytosis: enrichment of acanthocytic populations by density gradient centrifugation. J Lab Clin Med 1989; 113: 468-481.

9. Hardie RJ. Acanthocytosis and neurological impairment - a review. Quat J Med 1989; 264: 291-306.

10. Calne DB, Langston JW, Martin WRW, et al. Positron emission tomography after MPTP: observations relating to the cause of Parkinson's disease. Nature 1985; 317: 246-248.

11. Bruyn GW. Chorea-acanthocytosis. In: Vinken RJ, Bruyn GW, Klawans HL, eds. Extrapyramidal disorders. Handbook of Clinical Neurology; vol. 49. Amsterdam: Elsevier Science Publishers 1986; 327-334. 
12. Swash M, Schwartz MS, Carter ND, et al. Benign X-linked myopathy with acanthocytes (McLeod syndrome). Brain 1983; 106: 717-733

13. Jankovic J, Killian JM, Spitz MC. Neuroacanthocytosis syndrome and choreoacanthocytosis (Levine-Critchley syndrome). Neurology $1985 ; 35: 1679$.

14. Sakai T, Mawatari S, Iwashita $\mathrm{H}$, et al. Choreoacanthocytosis: clues to clinical diagnosis. Arch Neurol 1981; 38: 335-338.

15. Kondo K, Yanagisawa N. Familial chorea with neuropathy and acanthocytosis: report of six cases with special reference to its clinical variety. Neurol Med Chir (Tokyo) 1981; 15: 118-127.

16. Yamamoto T, Hirose G, Shimazaki K, et al. Movement disorders of familial neuroacanthocytosis syndrome. Arch Neurol 1982; 39: 298-301.

17. Ohnishi N, Sato Y, Nagara $\mathrm{H}$, et al. Neurogenic muscular atrophy and low density of large myelinated fibres of sural nerve in chorea-acanthocytosis. J Neurol Neurosurg Psychiatry 1981; 44: 645-648.
18. Limos LC, Ohnishi A, Sakai T, et al. "Myopathic" changes in chorea-acanthocytosis. J Neurol Sci 1982; 55: 49-58.

19. Spitz MC, Jankovic J, Killian JM. Familial tic disorder, parkinsonism, motor neuron disease, and acanthocytosis: a new syndrome. Neurology 1985; 35: 366-370.

20. Segawa M, Hosaka A. Miyagawa F, et al. Hereditary progressive dystonia with marked diumal fluctuation. Adv Neurol 1976; 14: 215-233.

21. Bird TD, Cederbaum S, Valpey RW, et al. Familial degeneration of the basal ganglia with acanthocytosis: a clinical, neuropathological and neurochemical study. Ann Neurol 1978; 3: 253-258.

22. Sato Y, Ohmishi A, Teteinshi J. An autopsy case of chorea-acanthocytosis. No To Shinkei 1984; 365: 105-111.

23. Spencer SE, Walker FO, Moore SA. Chorea-amyotrophy with chronic hemolytic anemia: a variant of chorea-acanthocytosis. Neurology 1987; 37: 645-649. 